\title{
TOPSiDE: Concept of an EIC Detector
}

\author{
José Repond ${ }^{* \dagger}$ \\ Argonne National Laboratory, 9700 S Cass Avenue, Lemont, IL 60439, U.S.A. \\ E-mail: repondeanl.gov
}

\begin{abstract}
We report on a detector concept, TOPSiDE, being developed for the EIC Electron-Ion Collider. TOPSiDE aims a the detection and identification of all particles created in electron-proton/ion collisions at the EIC while providing the best possible momentum/energy resolution. The measurement of hadronic jets exploits the advantages offered by Particle Flow Algorithms (PFAs), which in turn require imaging calorimetry with very fine granularity of the readout. Particle identification (pion-kaon-proton separation) is achieved through time-of-flight measurements in the tracker and the electromagnetic calorimeter, necessitating the application of ultra-fast silicon sensors. In the forward (hadron) direction particles are identified with a Čerenkov detector covering forward angles up to 10 degrees and a dipole or toroidal magnet for precise momentum measurements. The talk presents the detector concept, the status of its simulation software, first studies performed with a completed simulation tool chain, and the status of the detector R\&D related to the novel and challenging aspects of this concept detector.
\end{abstract}

XXVI International Workshop on Deep-Inelastic Scattering and Related Subjects (DIS2018) 16-20 April 2018

Kobe University Convention Centre / Kobe International Conference Center, Kobe, Japan

\footnotetext{
* Speaker.

${ }^{\dagger}$ on behalf of the TOPSiDE concept group.
} 


\section{Introduction: the Electron-Ion Collider}

The Electron-Ion Collider (EIC) is a planned facility to collide polarized electrons with polarized protons or ions with a center of mass energy between 35 and $140 \mathrm{GeV}$ and a luminosity of $10^{34} \mathrm{~cm}^{-2} \mathrm{~s}^{-1}$ [1]. Two possible sites are being considered: Brookhaven National Laboratory adding an electron ring to the existing RHIC machine and Thomas Jefferson National Laboratory utilizing the existing CEBAF facility for electron injection. The scientific goals of the EIC include the detailed study of both the perturbative and non-perturbative aspects of the theory of strong interactions, named Quantum Chromodynamics or QCD. Among other topics, the facility will perform a 3D imaging tomography of the nucleon and nuclei, i.e. the determination of parton densities as function of longitudinal momentum and transverse position or momentum. It will further our understanding of the nucleon spin by investigating the contributions from gluons and quark and gluon angular momenta. In addition, it will address a plethora of other topics, such as how the nucleon mass is generated or the possible saturation of the gluon density at low-x. The facility enjoys the unanimous support of the Nuclear Physics community and construction is slated for the latter part of the next decade.

\section{TOPSiDE: a 5D detector concept}

Several detector concepts, identified by names such as BEAST, JLEIC, ePHENIX and TOPSiDE [2], are being developed for the EIC. These concepts related to general purpose detectors attempt to address the entire, broad spectrum of physics topics to be studied at the EIC. Among these, the design philosophy of TOPSiDE is based on providing a hermetic detector, with the best possible energy/momentum resolution for single particles and complete particle identification, i.e. pion-kaon-proton separation. Utilizing such an outstanding performance, the detector will acquire events containing a list of identified particles and their momenta, rather than tracker hits and energy deposits in the calorimeter. In other words, each particle in the event will be identified and measured individually, ready for further physics analyses.

Following is a summary of the salient features of TOPSiDE: Unlike the colliding beam detectors at HERA (H1 and ZEUS) the central detector covering pseudorapidities of $-3<\eta<3$ is symmetric, driven by the notion that particles of all types are emitted into all directions. The vertex, outer, forward and backward trackers are based on silicon sensors. Calorimetry encloses the entire solid angle (hermetic) and features a very fine granularity of the readout. As is customary, the calorimeter is divided in depth into an electromagnetic and hadronic section, with each layer of both sections read out individually. As active medium the electromagnetic section utilizes silicon sensors with ultra-fast timing (for particle identification), while the hadronic section uses either scintillator pads with an area of order $3 \times 3 \mathrm{~cm}^{2}$ or Resistive Plate Chambers (RPCs) with $1 \times 1 \mathrm{~cm}^{2}$ pads and digital readout. Note that a concept which adds precision timing paired with extremely fine granularity of the readout to the measurement of energy is commonly named a 5D concept. The entire barrel calorimeter is placed inside a superconducting solenoid with a field of 2.5 Tesla. Placing the coil outside the calorimeter minimizes the amount of dead material in front of the calorimeter and thus avoids compromising its performance. 


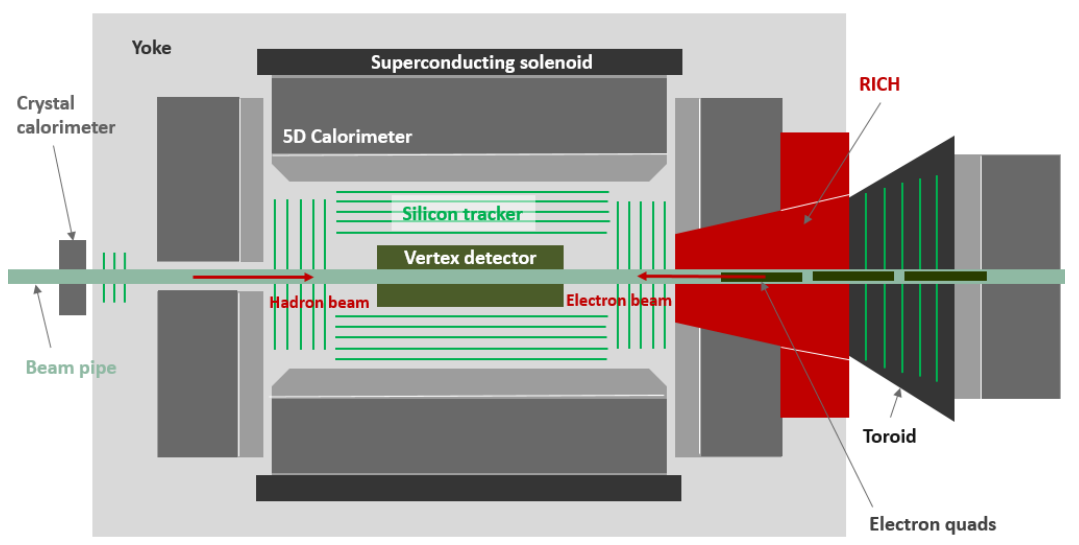

Figure 1: Sketch of the TOPSiDE concept.

In the forward (hadron) direction $(3<\eta<5)$ a gaseous ring imaging Čerenkov counter provides particle identification for momenta between 10 and $50 \mathrm{GeV} / \mathrm{c}$, whereas lower momenta particles are again identified through time-of-flight by ultra-fast silicon sensors in the forward tracking system. Precision momentum information is obtained with the help of a dipole or toroidal magnet in the forward direction. On the other side, in the backward (electron) direction $(-3<\eta<-5)$ a crystal calorimeter provides the best possible energy resolution for the scattered electron. Additional subsystems are integrated into the backward direction to tag low- $\mathrm{Q}^{2}$ and measure both the electron polarization and the luminosity. A sketch of the TOPSiDE concept is shown in Figure 1. Note that due to the 5D paradigm, no additional preshower, time-of-flight, or Čerenkov counters are needed in front of the calorimeter, which minimizes the amount of material between the event vertex and the calorimeter. Also, due to the imaging capability of the calorimeter, a muon system surrounding the detector becomes redundant.

In the following we provide additional details about the technologically challenging features of TOPSiDE, i.e. imaging calorimetry and ultra-fast silicon detectors.

\section{Imaging calorimetry}

Imaging calorimetry replaces the tower structure of traditional calorimeters with extremely fine segmentation of the readout, both laterally and longitudinally. Whereas typical calorimeters at colliding beam facilities count a few thousand channels, a $4 \pi$ imaging calorimeter features tens of millions of readout channels. Over the past decades, a number of technologies providing imaging capabilities have been investigated and validated: silicon sensors with areas of $0.16 \mathrm{~cm}^{2}$ to 1.00 $\mathrm{cm}^{2}$, scintillator strips with an area of $4.5 \times 0.5 \mathrm{~cm}^{2}$ for the electromagnetic section and scintillator pads with an area of $3 \times 3 \mathrm{~cm}^{2}$ and Resistive Plate Chambers (RPCs) with readout pads of $1 \times 1 \mathrm{~cm}^{2}$ for the hadronic section. Most of these studies have been performed by the CALICE collaboration [3] and members of the SiD detector concept [4]. As an example, Figure 2 shows an event display of a hadronic shower initiated by a $8 \mathrm{GeV}$ pion in the Digital Hadron Calorimeter (DHCAL). Note the visible, fine details of the hadronic shower with its emission of secondary charged particles.

Imaging calorimetry offers several distinct advantages over traditional calorimeters with tower structures: 


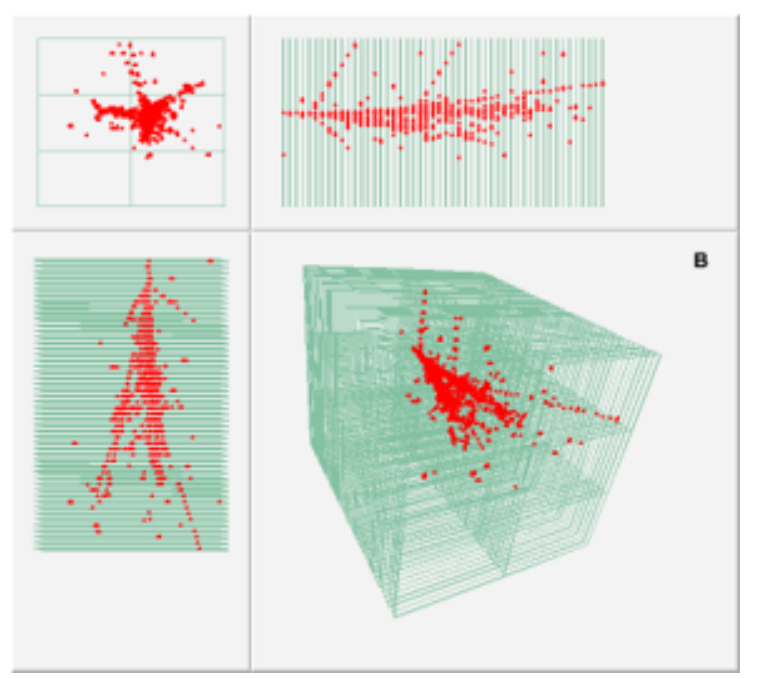

Figure 2: Event display of a hadronic shower initiated by a $8 \mathrm{GeV}$ pion in the Digital Hadron Calorimeter (DHCAL) with a Resistive Plate Chamber readout.

1. Particle identification. The identification of photons/electrons, muons, neutral and charged hadrons becomes trivial due to the precise measurement of shower shapes. For instance, a muon is seen as a minimum ionizing particle in every single layer of the calorimeter and can be identified as such even in a dense environment of other particles.

2. Software compensation. Typically the calorimetric response to same energy photons and pions is significantly different, with pions only registering a fraction of the amount of energy of electrons. With fine granularity of the readout electromagnetic subshowers in hadronic showers can be identified and their response can be weighted such as to equalize the response and therefore optimize the hadronic energy resolution [5]. This procedure is commonly referred to as software compensation.

3. Leakage corrections. Information about the longitudinal shower shape can be used to compensate for longitudinal leakage out the back of the calorimeter, again leading to an improvement of the hadronic energy resolution.

4. Gain monitoring. With the fine granularity of the readout, track segments within hadronic showers can be identified, see Figure 2, and utilized to monitor the gain of individual cells, thus providing a powerful, in situ calibration tool [6].

5. Identification of underlying events. Energy deposits from underlying events can be easily identified and subtracted from the event energy, due to the fine granularity of the readout. This advantage is more important at hadron colliders.

6. Application of Particle Flow Algorithms. Imaging calorimeters are able to measure each particle in a jet individually and are thus optimized for the application of Particle Flow Algorithms (PFAs) [7]. PFAs greatly improve the energy resolution of hadronic jets by using the subdetector providing the best energy/momentum measurement for each particle: charged 
tracks are measured with the tracking system, while neutrals (photons and neutral hadrons) are measured by the calorimeter.

TOPSiDE combines imaging calorimetry with precision timing measurement to provide pionkaon-proton separation. The timing measurement is based on ultra-fast silicon detectors, the topic of the next section.

\section{Ultra-fast silicon detectors}

TOPSiDE identifies hadrons (pions-kaons-protons) through time-of-flight measurements in the calorimeter and tracker. At the EIC, for most of the solid angle, hadron momenta are below 7 $\mathrm{GeV} / \mathrm{c}$, apart from the very forward direction where momenta can reach $50-100 \mathrm{GeV} / \mathrm{c}$ depending on the hadron beam energy. To identify hadrons with momenta up to $7 \mathrm{GeV} / \mathrm{c}$ and given the nominal inner radius of the electromagnetic calorimeter of say $120 \mathrm{~cm}$, timing resolutions of the order of 10 picosecond or better are needed (see next section).

The TOPSiDE concept uses ultra-fast silicon detectors (UFSD) [8] to achieve this challenging timing resolution. UFSD are being developed worldwide, mostly based on the Low-Gain Avalanche Detector (LGAD) technology [9]. Both reduction of the sensor thickness and the introduction of a thin amplification layer close to the n-p junction lead recently to significant improvements in the achievable timing resolution of silicon sensors [8]. Currently, the best performance is obtained with $35 \mu \mathrm{m}$ thick sensors with a timing resolution around 18 picosecond [10].

In collaboration with other institutions involved in the development of UFSD, Argonne National Laboratory is contributing to this effort on four fronts:

1. A test bench for silicon sensors has been assembled. The bench includes a high-bandwidth scope, low and high voltage power supplies, an environmental chamber etc.

2. Simulation of LGAD sensors has been initiated. The simulation utilizes the Silvaco software [11] and aims at the design of sensors with further improved timing resolution. A first iteration of a sensor design is shown in Figure 3. Next steps include the implementation of CMOS circuitry to perform the front-end processing of the signals.

3. Development of a time distribution system. Measuring times at the 10 picosecond level requires synchronization of the front-end electronics to at least the same degree of precision. The Argonne group is investigating the use of RF technology to achieve the required precision.

4. The laboratory is investing in a large scale sensor assembly facility, named AMAF for Argonne Micro Assembly Facility. The facility is expected to be commissioned early in 2019 and eventually will boast a complete set of tools for assembling and characterizing silicon sensors. 


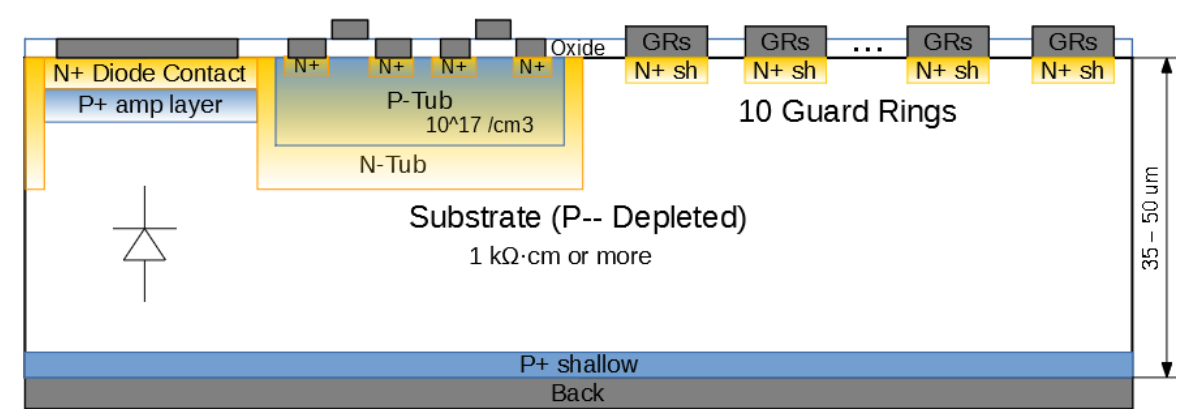

Figure 3: Design of a LGAD sensor with additional amplification layer close to the n-p junction.

\section{Simulation of the TOPSiDE detector}

A complete chain of simulation tools has been assembled to study the performance and optimize the design of TOPSiDE. The tool chain is based on GEANT4 [12] and includes event generation, particle transport through the detector, digitization of the detector response, track finding and fitting, particle identification, and event analysis. The geometry of the detector is defined in a sole source via DD4HEP [13] and was adapted from the SiD concept [4] originally developed for the International Linear Collider (ILC). Significant modifications from the original SiD concept were introduced, such as a longer barrel (motivated by the peculiarity of $e p$ scattering events), a lower magnetic field, and shallower calorimeters (motivated by the reduced center-of-mass energy of the EIC compared to the ILC), and a ring imaging Čerenkov counter coupled with a momentum analyzer in the forward direction. Figure 4 shows a display of an exclusive $\Upsilon$ event, as simulated in TOPSiDE.

Several studies were executed based on the complete simulation tool chain, investigating the energy resolution of single particles, the timing resolution requirement for time-of-flight identification of hadrons, reconstruction of kinematical variables, and last but not least the reconstruction of the $\mathrm{F}_{2}$ structure function and comparison to the one input into the generation of deep inelastic scattering events.

The simulation tool chain is general enough to be used for the simulation of any concept detector being considered for the EIC and other future experiments. The geometry being defined in a single source allows the dimensions of the detector to be parametrized and facilitates their adjustments. An interface to control version changes and execute automatic benchmark validations is being developed.

\section{Conclusions}

The TOPSiDE detector concept for the Electron-Ion Collider has been presented. It is in great part based on silicon sensors and features the 5D concept utilizing imaging calorimetry and ultrafast silicon sensors. The detector is completely hermetic and aims at providing a list of particles similar to the hadron level output of Monte Carlo simulations. Advantages of TOPSiDE include: the simplicity of the design (uniformity and limited number of subsystems), the use of silicon sensors (proven to be reliable, radiation hard, and requiring neither high voltages in excess of a $\mathrm{kV}$ 


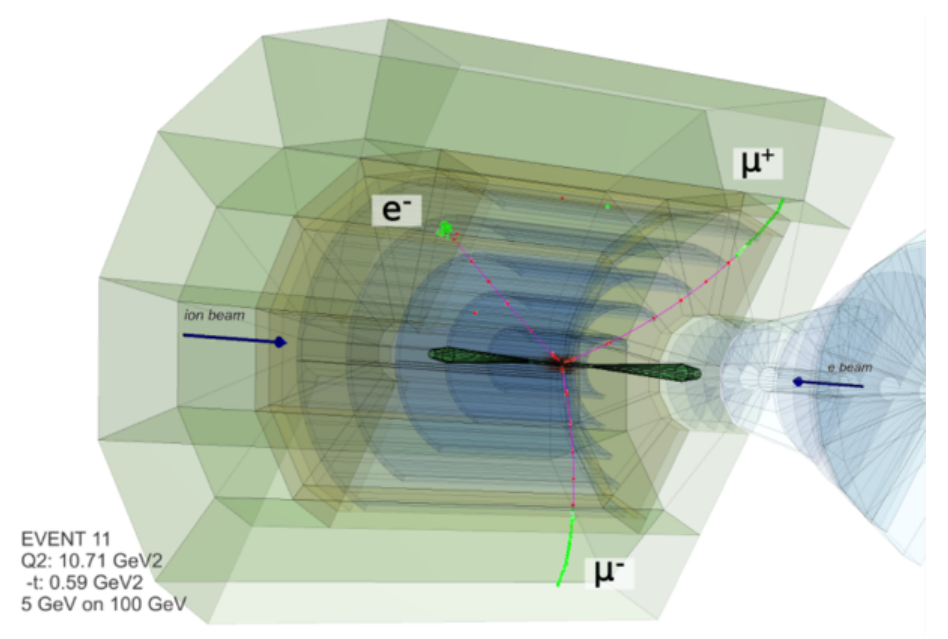

Figure 4: Event display of an exclusive $\Upsilon$ decaying into a pair of $\mu$ 's in the simulated TOPSiDE detector.

nor the supply of gas), excellent kinematic reconstruction, excellent background rejection, minimal inert material in front of the calorimeters, and no need for additional time-of-flight systems, $\breve{C}$ erenkov counters, or transition radiation detectors in front of the calorimeter, apart from a ring imaging C̆erenkov detector in the very forward direction.

\section{References}

[1] EIC White Paper: https://www.bnl.gov/npp/docs/EIC_White_Paper_Final.pdf

[2] "EIC Detectors: an Overview", talk given by J. Repond at the Electron-Ion Collider Users Group Meeting, Catholic University of America, Washington D.C.: https://www.jlab.org/conferences/eicugm18/

[3] CALICE collaboration: https://twiki.cern.ch/twiki/bin/view/CALICE/WebHome

[4] SiD concept: http://www.linearcollider.org/P-D/ILC-detector-concepts/SiD

[5] C. Adloff et al., JINST 7 (2012) P09017

[6] C. Adloff et al., JINST 8 (2013) P09001

[7] M.A. Thomson, Particle Flow Calorimetry and the PandoraPFA Algorithm, Nucl. Instrum. Meth. A611:25 (2009).

[8] H. Sadrozinski et al., Rep. Prog. Phys. 81 (2018) 026101 (34 pp)

[9] Workshop on pico-second timing detectors for physics and medical applications: https://agenda.infn.it/conferenceDisplay.py?confId=15031

[10] See H. Sadrozinski's talk in [9]

[11] Silvaco: https://www.silvaco.com/

[12] GEANT4: https://geant4.web.cern.ch/

[13] DD4HEP: https://github.com/AIDASoft/DD4hep 